\title{
Global Design of Perfect-Reconstruction Orthogonal Cosine-Modulated Filter Banks
}

\author{
Jie Yan and Wu-Sheng Lu \\ Department of Electrical and Computer Engineering \\ University of Victoria \\ Victoria, BC, Canada V8W 3P6 \\ jyan@ece.uvic.ca,wslu@ece.uvic.ca
}

\begin{abstract}
Designing perfect-reconstruction orthogonal cosinemodulated filter banks is essentially a nonconvex problem and to date only local solutions can be claimed. By virtue of the recent progress in global polynomial optimization, this paper describes an attempt of developing a global design method using an orderrecursive strategy combined with a technique that identifies a desirable initial point in each round of recursion. Simulation and comparison studies are presented to evaluate the performance of the proposed design technique.
\end{abstract}

\section{INTRODUCTION}

Orthogonal cosine modulated (OCM) filter banks are among the most popular filter banks for multirate signal processing as they admit efficient implementation through polyphase decomposition and their design can be carried out with considerably reduced complexity relative to that of a general $M$-channel system because in the former case one is focused on a single prototype filter (PF). A great deal of research on (locally) optimal design of OCM filter banks has been made [1]-[4], however, their global design remains a challenge primarily because the design problem is nonconvex.

This paper presents an attempt towards global design of OCM filter banks. In brief terms, the design is performed using an order-recursive strategy combined with a technique for identifying a desirable initial point in each round of the recursion. The recent breakthrough in global polynomial optimization problems (POPs) [5] is found useful in substantiating our design notion. Design examples are presented to illustrate the proposed method and compared with those obtained by existing design techniques.

\section{PRELIMINARIES}

An $M$-channel, maximally decimated OCM filter bank is illustrated in Fig. 1. The coefficients of the analysis and synthesis filters are respectively given by

$h_{k}(n)=2 h(n) \cos \left[\frac{\pi}{M}\left(k+\frac{1}{2}\right)\left(n-\frac{D}{2}\right)+(-1)^{k} \frac{\pi}{4}\right]$
$f_{k}(n)=2 h(n) \cos \left[\frac{\pi}{M}\left(k+\frac{1}{2}\right)\left(n-\frac{D}{2}\right)-(-1)^{k} \frac{\pi}{4}\right]$

for $0 \leq k \leq M-1$ and $0 \leq n \leq N-1$, where $\{h(n)\}$ is the impulse response of the finite-impulse-response (FIR) PF and $D$ is the system delay.

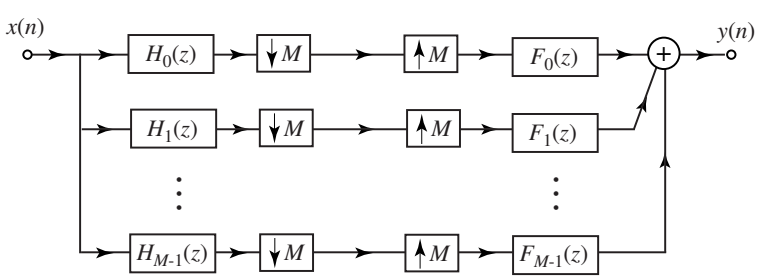

Fig. 1. $M$-channel maximally decimated filter bank.

There exist discrete cosine transform (DCT) modulations for OCM filter banks with structures other than that of (1) [1]. In this paper we concentrate on the DCT-IV OCM filter banks as defined in (1). In the rest of the paper, it is assumed that: 1) the channel number $M$ is even; 2) the filter length $N$ assumes the form $N=2 m M$ for some positive integer $m$; and 3) the system delay is $D=N-1$ since the PF of an OCM filter bank has a linear phase response. For the rationale of these assumptions, see [1]-[3].

The input-output relation of the system in the $z$-domain is given by

$$
Y(z)=T_{0}(z) X(z)+\sum_{l=1}^{M-1} T_{l}(z) X\left(z e^{-j 2 \pi l / M}\right)
$$

where

$$
T_{0}(z)=\frac{1}{M} \sum_{k=0}^{M-1} F_{k}(z) H_{k}(z)
$$

is the distortion transfer function determining the distortion caused by the system for the unaliased component $X(z)$, and

$T_{l}(z)=\frac{1}{M} \sum_{k=0}^{M-1} F_{k}(z) H_{k}\left(z e^{-j 2 \pi l / M}\right)$ for $l=1,2, \cdots, M-1$

are the alias transfer functions that determine how the aliased components $X\left(z e^{-j 2 \pi l / M}\right)$ are attenuated. The OCM filter bank holds the PR property if and only if

$$
T_{0}(z)=z^{-D}, \quad T_{l}(z)=0 \text { for } l=1,2, \cdots, M-1
$$

Under this circumstance, (2) becomes $Y(z)=z^{-D} X(z)$ and in the time domain the output is a delayed replica of the input as $y(n)=x(n-D)$. 
Typically, the "closeness" of an OCM filter bank to the PR property is measured in the frequency domain by means of

1) amplitude distortion

$$
e_{m}(\omega)=1-\left|T_{0}\left(e^{j \omega}\right)\right|, \quad \text { for } \omega \in[0, \pi]
$$

2) worst case aliasing error

$$
e_{a}(\omega)=\max _{1 \leq l \leq M-1}\left|T_{l}\left(e^{j \omega}\right)\right|, \quad \text { for } \omega \in[0, \pi]
$$

Alternatively, the PR conditions can be described in the time domain by the quadratic equations

$$
\begin{gathered}
a_{l, n}(\mathbf{h})=\mathbf{h}^{T} \mathbf{Q}_{l, n} \mathbf{h}-c_{n}=0 \\
\text { for } 0 \leq n \leq m-1 \text { and } 0 \leq l \leq M / 2-1
\end{gathered}
$$

where $\mathbf{h}=\left[\begin{array}{llll}h_{0} & h_{1} & \cdots & h_{N-1}\end{array}\right]^{T}$ denotes the coefficients of the $\mathrm{PF}$, and

$$
\begin{aligned}
\mathbf{Q}_{l, n} & =\mathbf{V}_{2 M-1-l} \mathbf{D}_{n} \mathbf{V}_{l}^{T}+\mathbf{V}_{M-1-l} \mathbf{D}_{n} \mathbf{V}_{M+}^{T} \\
\mathbf{D}_{n}(i, j) & = \begin{cases}1, & \text { if } i+j=n \\
0, & \text { otherwise }\end{cases} \\
\mathbf{V}_{l}(i, j) & = \begin{cases}1, & \text { if } i=l+2 j M \\
0, & \text { otherwise }\end{cases} \\
c_{n} & =\frac{1}{2 M} \delta(n-s)
\end{aligned}
$$

for $i=0,1, \cdots, N-1$ and $j=0,1, \cdots, N-1$.

We consider designing a PR OCM filter bank with its PF's stopband energy

$$
e_{2}(\mathbf{h})=\int_{\omega_{s}}^{\pi}\left|H\left(e^{j \omega}\right)\right|^{2} d \omega
$$

minimized, where $\omega_{s}=(1+\rho) \pi / 2 M$ is the stopband edge of the PF with $\rho>0$ ( $\rho$ is always assumed to be 1 in our designs). By writing

$$
H\left(e^{j \omega}\right)=\sum_{k=0}^{N-1} h_{k} e^{-j k \omega}=\mathbf{h}^{T} \mathbf{p}(\omega)
$$

with $\mathbf{p}(\omega)=\left[\begin{array}{llll}1 & e^{-j \omega} & \cdots & e^{-j(N-1) \omega}\end{array}\right]^{T}$ and using (8), the stopband energy becomes [4]

$$
e_{2}(\mathbf{h})=\mathbf{h}^{T} \mathbf{P h}
$$

where $\mathbf{P}$ is a symmetric positive-definite Toeplitz matrix determined by its first row $\left[\pi-\omega_{s},-\sin \omega_{s},-\sin 2 \omega_{s} / 2\right.$, $\left.\cdots,-\sin (N-1) \omega_{s} /(N-1)\right]$.

Since the impulse response of the PF is symmetrical, the design variables are only components in the first half of the PF's impulse response, i.e., $\hat{\mathbf{h}}=\left[h_{0} h_{1} \cdots h_{N / 2-1}\right]^{T}$. Thus, matrices $\mathbf{P}$ and $\mathbf{Q}_{l, n}$ of size $N \times N$ in (9) and (6a) are reduced to matrices $\hat{\mathbf{P}}$ and $\hat{\mathbf{Q}}_{l, n}$ of size $N / 2 \times N / 2$ and the optimization problem is cast as

$\operatorname{minimize} \quad e_{2}(\hat{\mathbf{h}})=\hat{\mathbf{h}}^{T} \hat{\mathbf{P}} \hat{\mathbf{h}}$

subject to: $\quad a_{l, n}(\hat{\mathbf{h}})=\hat{\mathbf{h}}^{T} \hat{\mathbf{Q}}_{l, n} \hat{\mathbf{h}}-c_{n}=0$

$$
\text { for } 0 \leq n \leq m-1 \text { and } 0 \leq l \leq M / 2-1
$$

The impulse response $\mathbf{h}$ can then be obtained as $\mathbf{h}=$ $\left[\hat{\mathbf{h}}^{T} \mathrm{flipud}(\hat{\mathbf{h}})^{T}\right]^{T}$ where flipud $(\hat{\mathbf{h}})$ denotes a vector generated by flipping vector $\hat{\mathbf{h}}$ upside down.

\section{GLOBAL DESIGN}

The design problem formulated in (10) is nonconvex because of the presence of the $m M / 2$ quadratic equality constraints in (10b). As a result, (10) admits local minimizers whose performance may be less than satisfactory. The principle idea we follow in this paper to reach a global design is to conduct a local search in a region that is sufficiently close to the global solution. To secure such a good starting (i.e. initial) point, we propose an order-recursive strategy based on the observation (see Sec. III-B) that the profile of the global solution of order $N=2 \mathrm{Mm}$ is quite close to that of the global solution of order $N^{\prime}=2 M^{\prime} m^{\prime}$ as long as the difference between $(m, M)$ and $\left(m^{\prime}, M^{\prime}\right)$ remains small. The design is accomplished by (i) obtaining a low-order global design and (ii) using interpolation/zero-padding of the design obtained to produce a desirable initial point for PF of slightly increased order and carry out the design by a locally optimal method. Repeat this step until the filter order reaches the targeted value. In what follows we describe the technical details that implement this design strategy.

\section{A. Global Design of Low-Order PFs}

Problem (10) is a polynomial optimization problem (POP) since the objective function and constraints are all polynomials of degree two. Reference [5] provides a semidefiniteprogramming [6] relaxation based method to obtain global solutions for a large class of POPs. GloptiPoly [7] and SparsePOP [8] are two popular solvers based on [5] in finding global solutions of POPs of small sizes. GloptiPoly was found to work well for problem (10). As an example, with $M=2$, $m=1$ and $\rho=1$, the toolbox produces two globally optimal impulse responses as

$$
\mathbf{h}^{(2,1)}=\left[\begin{array}{c}
\hat{\mathbf{h}}^{(2,1)} \\
\text { flipud }\left(\hat{\mathbf{h}}^{(2,1)}\right)
\end{array}\right]=\left[\begin{array}{l}
0.235923416966353 \\
0.440840267366581 \\
0.440840267366581 \\
0.235923416966353
\end{array}\right]
$$

and $-\mathbf{h}^{(2,1)}$. Unfortunately, the usefulness of the software is limited to OCM filter banks of low order. In our simulations the software was found to work only for the following cases: a) $M=2,1 \leq m \leq 5$; b) $M=4,1 \leq m \leq 3$; c) $M=6$, $m=1$; d) $M=8, m=1$.

\section{B. Global Design of High-Order PFs}

First, we observe a common pattern among globally optimal impulse responses of low-order PFs. Shown in Fig. 2 are the globally optimal impulse responses of low order obtained using GloptiPoly, where the impulse responses are plotted over normalized interval $[0,1]$. We note that:

1) For a fixed $M$, the impulse responses with different $m$ exhibit a similar pattern and are close to each other. 


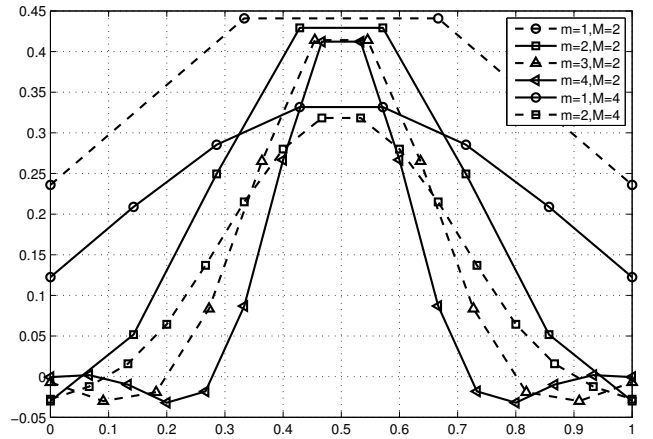

Fig. 2. Pattern of impulse responses of globally optimal PFs.

2) For $m=1$, the impulse responses with different $M$ also exhibit a similar shape.

These observations allow us to follow the design strategy described earlier which results in an algorithm as follows:

\section{Algorithm 1}

Input Data: Target filter order $\tilde{N}=2 \tilde{m} \tilde{M}$.

Step 1 Design a globally optimal PF with $m=1$ and $M=2$ using GloptiPoly. Denote the solution by $\mathbf{h}=$ $\left[\begin{array}{ll}\hat{\mathbf{h}}^{T} & \text { flipud }(\hat{\mathbf{h}})^{T}\end{array}\right]^{T}$.

Step 2 Design a globally optimal PF with $m=1$ and $M=\tilde{M}$ by the following steps:

2.1 Set $M=M+2$. Generate a vector $\hat{\mathbf{h}}_{0}^{\text {int }}$ of length- $(m M)$ by linear interpolating $\hat{\mathbf{h}}$, and obtain $\mathbf{h}_{0}^{\text {int }}$ by symmetrical extension of $\hat{\mathbf{h}}_{0}^{\text {int }}$.

2.2 Obtain a locally optimal design $\mathbf{h}$ with $\mathbf{h}_{0}^{\text {int }}$ as initial point (see Sec. IV).

2.3 If $M=\tilde{M}$, go to Step 3; otherwise, repeat from Step 2.1. Step 3 Design a globally optimal PF with $m=\tilde{m}$ and $M=$ $\tilde{M}$ by the following steps:

3.1 Set $m=m+1$. Generate a vector $\hat{\mathbf{h}}_{0}^{\text {zp }}$ of length $m \tilde{M}$ by padding zeros at the front of $\hat{\mathbf{h}}$, and obtain $\mathbf{h}_{0}^{\mathrm{zp}}$ by symmetrical extension of $\hat{\mathbf{h}}_{0}^{\mathrm{zp}}$.

3.2 Obtain a locally optimal design $\mathbf{h}$ with $\mathbf{h}_{0}^{\mathrm{zp}}$ as initial point (see Sec. IV).

3.3 If $m=\tilde{m}$, output $\mathbf{h}$ as the optimal design and terminate; otherwise, repeat from Step 3.1.

As an example, Fig. 3 illustrates Step 2.1, where the impulse response of the globally optimal PF with $m=1$ and $M=2$ is linearly interpolated to yield a vector $\mathbf{h}_{0}^{\text {int }}$ whose profile is similar to that of the globally optimal impulse response with $m=1$ and $M=4$. Another example is provided in Fig. 4 where vector $\mathbf{h}_{0}^{\mathrm{zp}}$ is produced by padding zeros to the globally optimal impulse response with $m=1$ and $M=4$. We see that $\mathbf{h}_{0}^{\mathrm{zp}}$ is quite close to the globally optimal impulse response with $m=2$ and $M=4$.

\section{LOCAL DESIGN}

Local design is an important ingredient of Algorithm 1 (see Steps 2.2 and 3.2). This section describes a sequential convex programming $(\mathrm{CP})$ technique for these local designs.

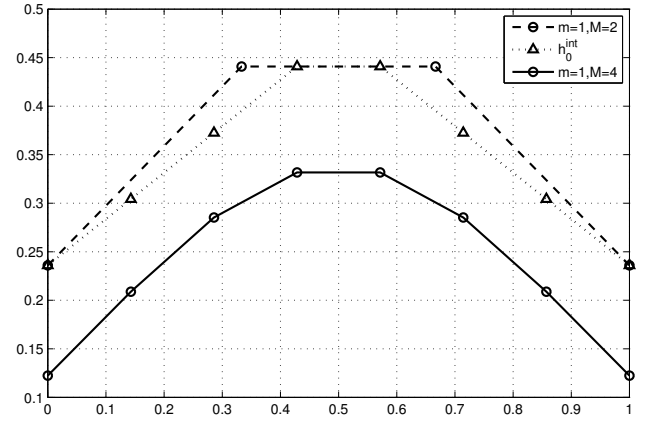

Fig. 3. Effect of linear interpolation when $m=1$.

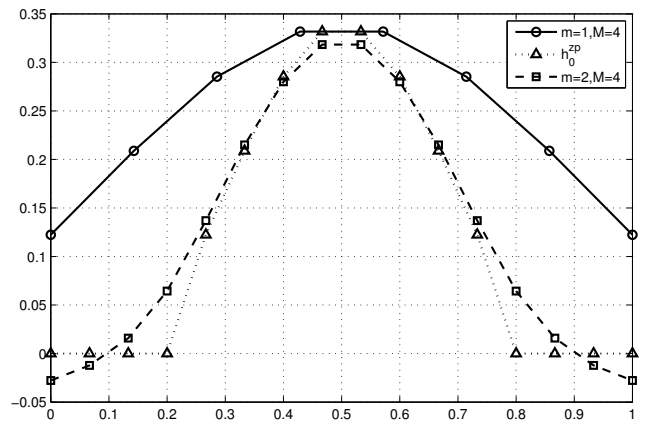

Fig. 4. Effect of zero-padding when $M=4$.

The design technique is in spirit similar to that proposed in [9], however here it is for a PF in OCM filter banks while [9] concerns with two-channel orthogonal filter banks and wavelets.

Suppose that we are in the $k$ th iteration to compute $\delta$ so that $\hat{\mathbf{h}}_{k+1}=\hat{\mathbf{h}}_{k}+\boldsymbol{\delta}$ reduces the PF's stopband energy in (10a) and better satisfies the PR conditions in (10b). The objective function in (10a) then becomes

$$
\hat{\mathbf{h}}_{k+1}^{T} \hat{\mathbf{P}} \hat{\mathbf{h}}_{k+1}=\boldsymbol{\delta}^{T} \hat{\mathbf{P}} \boldsymbol{\delta}+2 \boldsymbol{\delta}^{T} \hat{\mathbf{P}} \hat{\mathbf{h}}_{k}+\hat{\mathbf{h}}_{k}^{T} \hat{\mathbf{P}} \hat{\mathbf{h}}_{k}
$$

For $\boldsymbol{\delta}$ with $\|\boldsymbol{\delta}\|$ small, (10b) can be linearly approximated as

$$
\begin{aligned}
& a_{l, n}\left(\hat{\mathbf{h}}_{k}+\boldsymbol{\delta}\right) \approx a_{l, n}\left(\hat{\mathbf{h}}_{k}\right)+\mathbf{g}_{l, n}^{T}\left(\hat{\mathbf{h}}_{k}\right) \boldsymbol{\delta}=0 \\
& \text { for } 0 \leq n \leq m-1 \text { and } 0 \leq l \leq M / 2-1
\end{aligned}
$$

where $\mathbf{g}_{l, n}\left(\hat{\mathbf{h}}_{k}\right)=2 \hat{\mathbf{Q}}_{l, n} \hat{\mathbf{h}}_{k}$ is the gradient of $a_{l, n}(\hat{\mathbf{h}})$ at $\hat{\mathbf{h}}_{k}$. Using (11) and (12), the $k$ th iteration of (10) assumes the form

$$
\begin{aligned}
\operatorname{minimize} & \boldsymbol{\delta}^{T} \hat{\mathbf{P}} \boldsymbol{\delta}+\boldsymbol{\delta}^{T} \mathbf{b}_{k} \\
\text { subject to: } & \mathbf{G}_{k} \boldsymbol{\delta}=-\mathbf{a}_{k} \\
& \|\boldsymbol{\delta}\| \text { is small }
\end{aligned}
$$

where $\mathbf{b}_{k}=2 \hat{\mathbf{P}} \hat{\mathbf{h}}_{k}, \mathbf{G}_{k} \in R^{N / 4 \times N / 2}$ collects the $N / 4$ rows $\mathbf{g}_{l, n}^{T}\left(\hat{\mathbf{h}}_{k}\right)$ and $\mathbf{a}_{k} \in R^{N / 4}$ consists of $N / 4$ components $a_{l, n}\left(\hat{\mathbf{h}}_{k}\right)$. The equality constraint (13b) can be eliminated via the singular value decomposition of $\mathbf{G}_{k}=\mathbf{U} \boldsymbol{\Sigma} \mathbf{V}$ as [6]

$$
\boldsymbol{\delta}=\mathbf{V}_{e} \boldsymbol{\phi}+\boldsymbol{\delta}_{s}
$$


Assuming $\mathbf{G}_{k}$ has full row-rank that is $N / 4$, then $\phi$ is an $N / 4$-dimensional free vector, $\mathbf{V}_{e}$ is a matrix consisting of the last $N / 4$ columns of $\mathbf{V}$, and $\boldsymbol{\delta}_{s}=-\mathbf{G}_{k}^{\dagger} \mathbf{a}_{k}$ where $\dagger$ denotes the pseudo-inverse of a matrix. Thus, (13) can be cast as

$$
\begin{aligned}
\text { minimize } & \phi^{T} \tilde{\mathbf{P}}_{k} \boldsymbol{\phi}+\boldsymbol{\phi}^{T} \tilde{\mathbf{b}}_{k} \\
\text { subject to: } & \|\boldsymbol{\phi}\| \text { is small }
\end{aligned}
$$

where $\tilde{\mathbf{P}}_{k}=\mathbf{V}_{e}^{T} \hat{\mathbf{P}} \mathbf{V}_{e}, \tilde{\mathbf{b}}_{k}=2 \mathbf{V}_{e}^{T} \hat{\mathbf{P}}\left(\hat{\mathbf{h}}_{k}+\boldsymbol{\delta}_{s}\right)$. Since (15) is a convex QP problem, it can be solved efficiently in MATLAB using command quadprog ( ). Using the solution of (15) in Eq. (14), an optimal $\boldsymbol{\delta}_{k}$ is obtained and point $\hat{\mathbf{h}}_{k}$ is updated to point $\hat{\mathbf{h}}_{k+1}$. This iterative procedure continues until $\left\|\boldsymbol{\delta}_{k}\right\|$ is less than a prescribed tolerance.

We now conclude this section with a couple of remarks. 1. As can be seen in (15b), the constraint on the smallness of $\|\boldsymbol{\delta}\|$ (see (13c)) is replaced by a constraint on the smallness of $\|\phi\|$. This change is justified by noticing that (14) implies that $\|\boldsymbol{\delta}\| \leq\|\phi\|+\left\|\boldsymbol{\delta}_{s}\right\|$ where $\boldsymbol{\delta}_{s}$ is a minimum-norm solution of (13b), hence a sufficiently small $\|\boldsymbol{\phi}\|$ implies a small $\|\boldsymbol{\delta}\|$. 2 . The algorithm occasionally does not converge to a highly accurate solution because of numerical difficulties. In such a case, the filter coefficients obtained are slightly adjusted using a Gauss-Newton technique [6] with adaptively controlled weights in order for the final solution to satisfy the PR condition (10b) with high accuracy.

\section{SIMULATION RESULTS}

\section{A. Performance of Algorithm 1 for Low-Order Designs}

The design strategy described in Sec. III-B was applied to design low-order PFs for the cases: a) $M=2,2 \leq m \leq 5$; b) $M=4,1 \leq m \leq 3$; c) $M=6, m=1$; d) $M=8, m=1$. The impulse responses obtained were found to be practically identical to those generated from GloptiPoly in Sec. III-A, giving a convincing support to our design concept.

\section{B. Performance of Algorithm 1 for High-Order Designs}

The proposed method was also applied to design highorder PFs of OCM filter banks with satisfactory results. As an example, an OCM filter bank with $m=20$ and $M=4$ was designed using Algorithm 1. The design was performed on a PC laptop with a $1.66 \mathrm{GHz}$ dual-core processor. The magnitude response of the optimized PF is shown in Fig. 5. For comparison, an OCM filter bank with the same design specifications was designed by the method in [4]. Table I lists the performance of the two filter banks in terms of stopband energy (see (10a)) of the optimized PFs and the largest equation error amongst all quadratic equality constraints in (10b). To explicitly measure the PR satisfaction, Table I also lists the maximum of absolute value of amplitude distortion (see Eq. (4)) as well as the maximum of absolute value of worst case aliasing error (see Eq. (5)), for $\omega \in[0, \pi]$. From the table, the superior performance of the filter bank produced by the proposed design technique is observed. We remark however that the performance gain was at the cost of increased computational complexity: the proposed design required 59.5

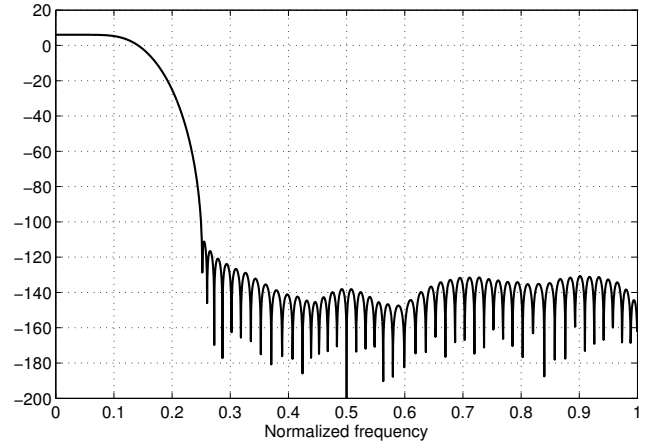

Fig. 5. Magnitude response of globally optimal PF of an OCM filter bank with $m=20, M=4$ and $\rho=1$.

TABLE I

PERFORMANCE COMPARISON

\begin{tabular}{|c|c|c|}
\hline & Global design & Local design \\
\hline Energy in stopband & $8.226 \mathrm{e}-13$ & $6.585 \mathrm{e}-10$ \\
\hline Largest eq. error & $1.839 \mathrm{e}-15$ & $2.297 \mathrm{e}-10$ \\
\hline $\max \left(\left|e_{m}(\omega)\right|\right)$ & $3.975 \mathrm{e}-14$ & $9.811 \mathrm{e}-10$ \\
\hline $\max \left(\left|e_{a}(\omega)\right|\right)$ & $3.314 \mathrm{e}-14$ & $3.003 \mathrm{e}-9$ \\
\hline
\end{tabular}

seconds of CPU time versus 19.7 seconds by the method in [4].

\section{CONCLUSION}

A strategy for the global design of PR OCM filter banks has been proposed. The method is built upon some recent progress in global polynomial optimization and an effective local design technique, in conjunction with several critical observations on the globally optimal impulse responses. Several design examples have been presented to verify the design concept and demonstrate the superiority of the proposed algorithm.

\section{REFERENCES}

[1] P. N. Heller, T. Karp, and T. Q. Nguyen, "A general formulation of modulated filter banks," IEEE Trans. Signal Processing, vol. 47, pp. 9861002, Apr. 1999.

[2] T. Karp, A. Mertins, and G. Schuller, "Efficient biorthogonal cosinemodulated filter banks," Signal Processing, vol. 81, pp. 997-1016, May. 2001.

[3] T. Saramäki and R. Bregović, "Multirate systems and filter banks," in Multirate Systems: Design and Applications, G. Jovanovic-Dolecek, Ed. Hershey, PA: Idea Group, 2002, ch. 2.

[4] W.-S. Lu, T. Saramäki and R. Bregović, "Design of practically perfectreconstruction cosine-modulated filter banks: a second-order cone programming approach," IEEE Trans. Signal Processing, vol. 51, pp. 552563, Mar. 2004.

[5] J. B. Lasserre, "Global optimization with polynomials and the problem of moments," SIAM J. Optimization, vol. 11, no. 3, pp. 796C817, 2001.

[6] A. Antoniou and W.-S. Lu, Practical Optimization: Algorithms and Engineering Applications, Springer, 2007.

[7] D. Henrion, J. B. Lasserre, and J. Löfberg, "GloptiPoly 3: moments, optimization and semidefinite programming," LAAS-CNRS Report, Toulouse, France Sep 2008.

[8] H. Waki, S. Kim, M. Kojima, and M. Muramatsu, "SparsePOP: A sparse semidefinite programming relaxation of polynomial optimization problems," Research Report, Tokyo Institute of Technology, March 2005.

[9] W.-S. Lu and T. Hinamoto, "Direct Design of Orthogonal Filter Banks and Wavelets," ISCAS 2009, pp. 81-84, Taipei, Taiwan, May 24-27, 2009. 